\title{
Shear Melting of a Colloidal Glass
}

\section{Citation}

Eisenmann, Christoph, Chanjoong Kim, Johan Mattsson, and David A. Weitz. 2010. "Shear Melting of a Colloidal Glass." Physical Review Letters104 (3): 035502. https://doi.org/10.1103/ PhysRevLett.104.035502.

\section{Permanent link}

http://nrs.harvard.edu/urn-3:HUL.InstRepos:41511251

\section{Terms of Use}

This article was downloaded from Harvard University's DASH repository, and is made available under the terms and conditions applicable to Other Posted Material, as set forth at http:// nrs.harvard.edu/urn-3:HUL.InstRepos:dash.current.terms-of-use\#LAA

\section{Share Your Story}

The Harvard community has made this article openly available.

Please share how this access benefits you. Submit a story.

\section{Accessibility}




\title{
Shear Melting of a Colloidal Glass
}

\begin{abstract}
Christoph Eisenmann, Chanjoong Kim, ${ }^{*}$ Johan Mattsson, ${ }^{\dagger}$ and David A. Weitz
Department of Physics and HSEAS, Harvard University, Cambridge, Massachusetts 02138, USA (Received 29 August 2008; revised manuscript received 1 December 2009; published 21 January 2010)

We use confocal microscopy to explore shear melting of colloidal glasses, which occurs at strains of $\sim 0.08$, coinciding with a strongly non-Gaussian step size distribution. For larger strains, the particle mean square displacement increases linearly with strain and the step size distribution becomes Gaussian. The effective diffusion coefficient varies approximately linearly with shear rate, consistent with a modified Stokes-Einstein relationship in which thermal energy is replaced by shear energy and the length scale is set by the size of cooperatively moving regions consisting of $\sim 3$ particles.
\end{abstract}

Colloidal suspensions provide a valuable model system for the study of the glass transition [1]. They exhibit many properties that mimic the behavior of more traditional molecular glass formers; moreover, the large particle size and the slow dynamics makes it possible to use direct imaging techniques to study the behavior of individual particles. This provides considerable new insight into features that are ubiquitous to the glass transition such as cooperativity and dynamic heterogeneity $[2,3]$. However, colloidal glasses also possess features that are unique to them. Perhaps the most remarkable is shear melting. Because of the large particle size, colloids form soft solids; thus they can be fluidized through shear [4], which cannot be easily done for molecular glasses. Shear melting helps account for the widespread utility of these systems, since they can be solidlike under quiescent conditions, but can flow like a fluid when sheared. Such shear-induced fluidization is one means of driving a solid-to-fluid transition within the generic jamming "phase" diagram [5], wherein strong similarities should exist among shear-induced [6], concentration-induced, and temperature-induced fluidization. However, a full understanding of the relationship of shear-induced fluidization to the other, more common methods of fluidization requires a detailed understanding of the microscopic behavior of shear melting; this remains elusive.

In this Letter, we explore the microscopic nature of shear melting of colloidal glasses by using confocal microscopy to measure the fluctuations of particle motion beyond the average imposed strain. We find that shear melting induces cooperative motion, extending over 3 particles on average, provided a strain, $\gamma \approx 0.08$, is exceeded. At large strains, the mean square displacement (MSD) of the particles increases linearly with strain and the distribution of step sizes becomes Gaussian. Remarkably, this diffusive behavior can be accounted for by a modified Stokes-Einstein expression, where thermal energy is replaced by shear energy and the length scale is set by the size of the cooperative regions.

We investigate a colloidal suspension of poly(methyl methacrylate) particles with an average radius $R=$
$0.6 \mu \mathrm{m}$ and a polydispersity of $\sim 4 \%$. The particles are sterically stabilized with a thin layer of poly(hydroxy stearic acid) and are fluorescently labeled with nitrobenzoxadiazole. They are suspended in a mixture of cisdecalin and cycloheptylbromide, which matches both the particle density and index of refraction. To minimize the effects of charge on the particles, we add $1 \mathrm{~g} / \mathrm{L}$ of the salt tetrabutylammonium chloride [7]. The volume fraction is $\phi=0.61 \pm 0.03$; this is a colloidal glass, as confirmed by its nonergodic behavior probed by dynamic light scattering. The suspension is contained between two parallel glass plates separated by a $40 \mu \mathrm{m}$ gap in a specially designed air-tight shear cell. The surfaces of the plates are roughened by a sintered coating of polydisperse particles (average radius is $1.5 \mu \mathrm{m}$ and polydispersity $\sim 30 \%$ ) to avoid slip at the surface and to inhibit surface-induced ordering. We observed a linear shear gradient throughout the sample and did not detect any sign of crystallinity.

We follow the motions of individual colloidal particles under shear using confocal laser scanning microscopy; the particle positions are tracked as the suspension is subjected to shear. We focus the analysis on the central region of the suspension to avoid wall effects and restrict the analysis to a two-dimensional plane to allow us to follow the rapid motion of individual particles. A symmetrical triangular time-dependent strain is applied to the sample in the $y$ direction with strain amplitudes up to $\gamma \approx 0.5$ and strain periods varying between 25 and $100 \mathrm{~s}$, resulting in shear rates between 0.003 and $0.02 \mathrm{~s}^{-1}$. The Péclet number, which determines the ratio of the shear rate to the rate of diffusion, is less than 0.1 if the self-diffusion coefficient of dilute particles, $D_{0}=0.14 \mu \mathrm{m}^{2} / \mathrm{s}$, is used. However, because we are investigating particles' motions that are comparable to their size, the diffusion coefficient should more properly be chosen as the long-time diffusion coefficient, which reflects the structural relaxation of the particles and is many orders of magnitude smaller than $D_{0}$. As a result the effective Péclet number is very large and the motion is dominated by the imposed shear [8]. To ensure reproducible results, the sample is subjected to strain for many periods at the chosen strain rate, after which data are 
collected for ten cycles at two frames per second. We track the position of each particle in the two-dimensional plane and determine its position $x(t), y(t)$ after subtraction of the mean displacement due to the applied shear.

To investigate initiation of shear melting, we take advantage of the periodic application of strain and calculate an effective MSD, $\left\langle\Delta x^{2}(\Delta t)\right\rangle_{0}=\left\langle\left(x\left(t_{0}+\Delta t\right)-x\left(t_{0}\right)\right)^{2}\right\rangle$, where $t_{0}$ is always taken as the initial time of a half-cycle and the averaging is repeated over 10 shear cycles. The behaviors in the $x$ and $y$ directions are very similar, but the $y$ component is about 30\%-50\% larger, consistent with previous results $[9,10]$ and presumably reflecting the anisotropy expected as a result of Taylor dispersion [11]. Since the qualitative behavior is so similar, here we focus only on the $x$ component, $\left\langle\Delta x^{2}(\Delta t)\right\rangle_{0}$.

Since the motion is driven by the strain, we plot the MSD as a function of the strain accumulated during the lag time $\Delta t$, allowing us to compare the data obtained at all shear rates. In all cases the behavior of the MSD is nearly identical: For $\gamma<0.08$, subdiffusive behavior is observed; this is followed by a crossover to a diffusive regime, as shown in Fig. 1(a). This behavior is reminiscent of that found for materials that shear melt at a critical strain [4]. To explore whether our results are consistent with such behavior, we calculate the MSD averaged over start times $t_{y}$ chosen after the onset of diffusive behavior, corresponding to $\gamma>0.08,\left\langle\Delta x^{2}(\Delta t)\right\rangle_{y}=\left\langle\left(x\left(t_{y}+\Delta t\right)-x\left(t_{y}\right)\right)^{2}\right\rangle$. As shown in Fig. 1(b), we find a similar behavior for every start time implying that the observed behavior does not reflect a critical strain. This result is consistent with previous findings of both "in-cage" rattling motions [10] and

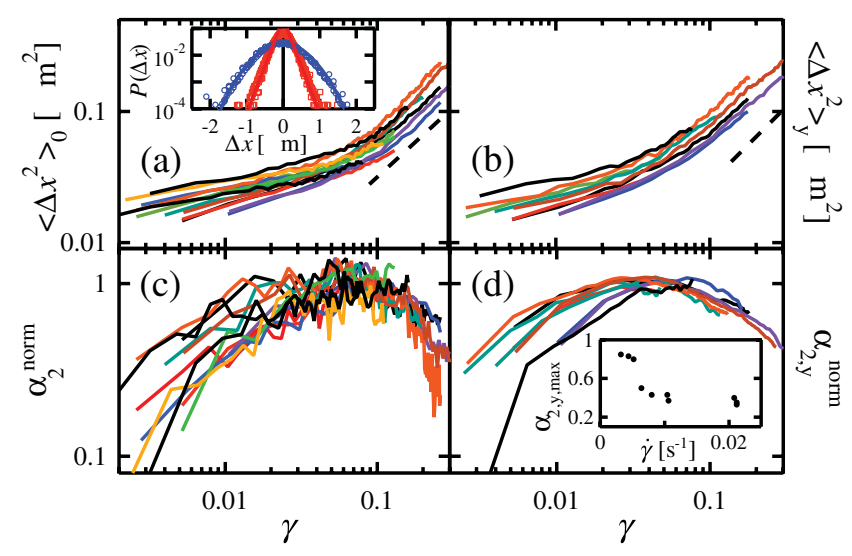

FIG. 1 (color online). (a) $\left\langle\Delta x^{2}(\Delta t)\right\rangle_{0}$ vs $\gamma$ for a sheared colloidal glass for $\dot{\gamma}$ varying from 0.003 to $0.02 \mathrm{~s}^{-1}$. Inset: Distribution function $P(\Delta x(\Delta t))$ for the sheared glass $\left(\dot{\gamma}=0.02 \mathrm{~s}^{-1}\right)$ for $\Delta t=4 \mathrm{~s}$ [(red) squares] and $\Delta t=50 \mathrm{~s}$ [(blue) circles], together with an exponential and a Gaussian fit, respectively. (b) $\left\langle\Delta x^{2}(\Delta t)\right\rangle_{y}$ vs $\gamma$, calculated with a start time always chosen in the "yielded" regime. (c) $\alpha_{2}$, as calculated from the data in (a) as a function of $\gamma ; \alpha_{2}$ is normalized by its maximum value. (d) Normalized $\alpha_{2, y}$ calculated from the data in (b). Inset: Maximum of $\alpha_{2, y}$ as a function of $\dot{\gamma}$. a significant elastic contribution [4,12] for shear-melted collolidal hard sphere glasses at strains well above $\gamma \sim$ $0.07-0.15$.

The distribution of particle displacements in the liquid state of a colloidal suspension is Gaussian. By contrast, as the glass transition is approached, strongly non-Gaussian behavior is observed reflecting the existence of dynamic heterogeneities [13]. To examine the behavior of a shearmelted glass, we determine the displacement distribution function $P(\Delta x(\Delta t))$. For large lag times, corresponding to $\gamma>0.08$, the distribution is Gaussian, consistent with liquidlike dynamics; by contrast, for short lag times corresponding to $\gamma \sim 0.08$, the distribution is strongly nonGaussian and is instead better described as exponential, as shown in the inset of Fig. 1(a). This suggests that the motion is highly heterogeneous at small strains, but becomes more homogeneous at large strains.

We can further quantify the observed behavior by calculating the non-Gaussian parameter $\alpha_{2}(t)=\frac{\left\langle\Delta x^{4}\right\rangle}{3\left\langle\Delta x^{2}\right\rangle^{2}}-1$, which is zero for Gaussian dynamics and nonzero otherwise. To compare the data, we normalize $\alpha_{2}$ for each data set by its maximum. Independent of the starting time, we find identical behavior for the normalized $\alpha_{2}$; it reaches a maximum at $\gamma \sim 0.08$ independent of $\dot{\gamma}$, as shown in Figs. 1(c) and 1(d). This maximum corresponds to the crossover between subdiffusive and diffusive regimes in the MSD. Similar behavior is observed for a colloidal liquid near its glass transition [3], where $\alpha_{2}$ reaches a maximum at the onset of structural relaxation and particle flow. Moreover, the maximum value of $\alpha_{2}$ decreases with $\dot{\gamma}$, but reaches a plateau at high $\dot{\gamma}$ as shown in the inset of Fig. 1(d); a qualitatively similar behavior has been observed in [10].

To explore the nature of the non-Gaussian behavior, we calculate the two-point correlation function $D_{r r}(r, \Delta t)=$ $\left\langle\Delta r^{i}(\Delta t) \Delta r^{j}(\Delta t)\right\rangle_{\left|r^{i}-r^{j}\right|=r}$, where $\Delta r^{i}(\Delta t)$ is the projection of the displacement onto the vector between the two particles [14]. For any continuum material either fluid or solid $D_{r r}(r, \Delta t) \sim 1 / r$; a deviation from this behavior is direct evidence of anomalous particle dynamics. We therefore calculate $r D_{r r}$, to sensitively explore the range of the $1 / r$ behavior. For long delay times the data follow a straight line with zero slope, as shown in Fig. 2(a). However, as the delay time decreases, the data instead exhibit an increasingly sharp decay. These results suggest the onset of a shear-induced behavior. To quantify this behavior we calculate the ratio of $r D_{r r}$ measured at $r=10 \mu \mathrm{m}$ and $r=3 \mu \mathrm{m}$; a value of 1 corresponds to $D_{r r} \sim 1 / r$, whereas a smaller value indicates faster decay. We plot this ratio as a function of $\gamma$ and find an exponential growth from 0 to a value near 1 , as shown in Fig. 2(b). An exponential fit gives a characteristic strain $\gamma \approx 0.08$, in accord with the strain amplitude where the MSD crosses over from subdiffusive to a steady state diffusive behavior. 


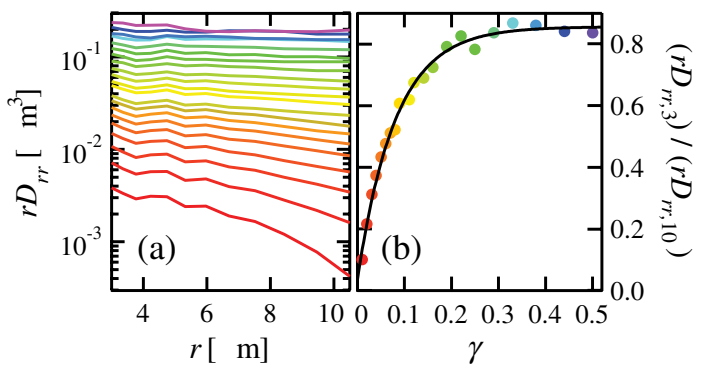

FIG. 2 (color online). (a) Two-point correlation function $D_{r r}$, multiplied by $r$ for a sheared glass $\left(\dot{\gamma}=0.02 \mathrm{~s}^{-1}\right)$ for different lag times ranging from $\Delta t=0.5 \mathrm{~s}$ [bottom (red) line] to $\Delta t=$ $25 \mathrm{~s}$ [top (purple) line]. (b) Ratio of $r D_{r r}$ at $r=10 \mu \mathrm{m}$ and $r=$ $3 \mu \mathrm{m}$ vs $\gamma$. The line is an exponential fit with a characteristic strain $\gamma_{c}=0.08$.

To explore the nature of cooperative motions in the sheared sample, we quantify the range of cooperativity in the particle motions. We focus on particles that move further than a threshold distance $\delta$ within a lag time $\Delta t$ corresponding to a fixed accumulated strain in the diffusive regime. We find that the particular choice of strain and threshold distance is not critical and any reasonable choice of parameters characterizing the diffusive regime gives very similar results; typically, we use a threshold distance $\delta \simeq 0.3 \mu \mathrm{m} \simeq R / 2$ and $\gamma \simeq 0.15$. We color-code the particles with respect to their direction of movement: Particles

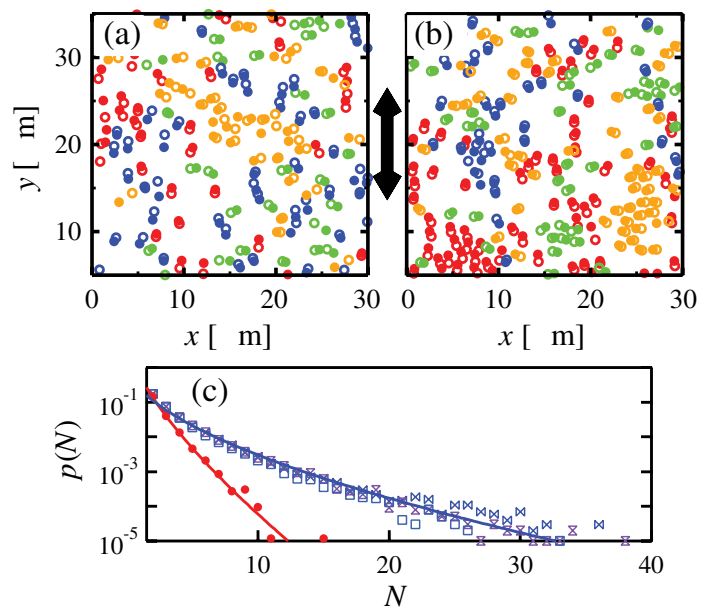

FIG. 3 (color online). Dynamic clusters for a low-volume fraction, $\sim 40 \%$, liquid (a) and a sheared glass with $\dot{\gamma}=0.01 \mathrm{~s}^{-1}$ (b). The full symbols indicate particle positions at $t=t_{0}$, the open symbols positions at $t=t_{0}+15 \mathrm{~s}$, corresponding to $\gamma=0.15$. Red (dark gray) and blue (black): Moving in $+/-y$ direction. Green (gray) and orange (light gray): Moving in $+/-x$ direction. The sample is sheared in the $y$ direction. For clarity, particle sizes are not drawn to scale. (c) Size histogram for the dynamic clusters of the shear-melted glass (blue empty symbols) for various $\dot{\gamma}$ 's from 0.008 to $0.02 \mathrm{~s}^{-1}$, at the same $\gamma=0.15$ and the unsheared low-volume fraction liquid (red filled circles). The lines are guides to the eye. with a large displacement component in the positive $x$ direction, $\Delta \vec{r}(\Delta t) \cdot \hat{x} / \Delta r \geq 0.8$ and $\Delta r(\Delta t) \geq \delta$, are colored in green (gray); analogously orange (light gray) is used for motions in the $-x$ while red (dark gray) and blue (black) are used for the $+y$ and $-y$ directions, respectively. We compare the cooperative particle motions of a shearmelted glass to those of a low $\phi(\sim 40 \%)$ colloidal liquid with a comparable diffusion coefficient. Snapshots of the particle motions as shown in Fig. 3 document the development of larger clusters for the shear-melted glass than for the unsheared low-volume fraction liquid. Interestingly, we observe clusters of particles moving both parallel and perpendicular to the direction of the applied strain.

To quantitatively compare the average dynamic cluster size for the low-volume fraction liquid and the sheared glass, we plot the dynamic cluster size distribution for each sample. Two particles of the same color were assigned to the same dynamic cluster if the interparticle distance was less than the first minimum in the distinct radial distribution function. We find that the sheared glass has a longer tail of larger dynamic clusters, as shown in Fig. 3(c). Integrating the data, we obtain an average dynamic cluster size of about 2-3 particles, setting the scale of the cooperative motion. We do not find any shear-rate dependence of this size within the accuracy of the measurements. This size is in good agreement with the measurements of shear transformation zones observed in a colloidal glass subjected to small strains and low shear rates [15].

A further comparison between the fluid sample and the shear-melted sample is obtained by measuring the shearinduced diffusion coefficient for the particle motion. We calculate the MSDs for the shear-melted sample as $\left\langle\Delta x^{2}(t)\right\rangle_{y}$ and find that the MSDs are linear in time as shown in Fig. 4(a). From linear fits to the data, we extract the long-time diffusion constants $D$ of the shear-melted glass from $\left\langle\Delta x^{2}(t)\right\rangle_{y}=2 D t$. The increase of the diffusion constant $D$ with $\dot{\gamma}$ is consistent with a linear behavior, as shown in Fig. 4(b). A recent study [10] on a similar shear-

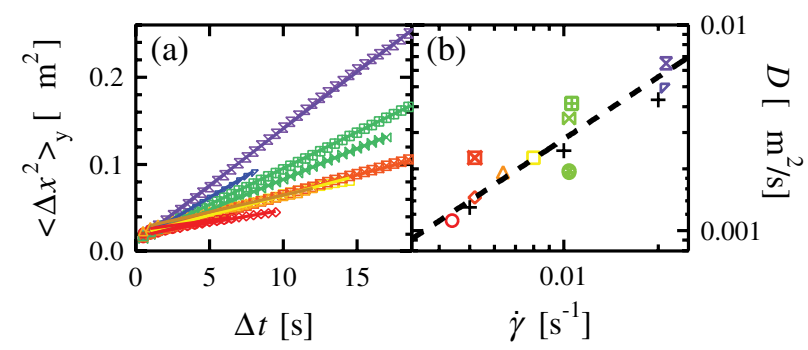

FIG. 4 (color online). (a) $\left\langle\Delta x^{2}(\Delta t)\right\rangle_{y}$ as a function of lag time $\Delta t$ for a shear-melted glass at different applied shear rates. The lines are linear fits to the data. (b) Long-time diffusion coefficient $D$, extracted from the linear fits in (a), as a function of shear rate $\dot{\gamma}$. The symbols correspond to those of the data in (a) except the + symbols, showing data taken from Ref. [19]. The dashed line corresponds to $D=\frac{2}{9} R_{s}{ }^{2} \dot{\gamma}$, with $R_{s}=1.8 R ; R$ is the particle radius. 
melted hard sphere colloidal glass instead suggests $D \propto$ $\dot{\gamma}^{0.8}$. However, these samples exhibited shear banding albeit somewhat away from the studied region; by contrast, shear banding is never observed in our samples. These different shear profiles may lead to the difference in the $\dot{\gamma}$ dependence of $D$. Similarly, theoretical predictions for $D$ suggest that $D$ scales as $\dot{\gamma}[8,16]$; other theoretical approaches suggest similar scaling through calculation of structural relaxation times [17], although one suggests a lower exponent [18]. Moreover, a behavior highly similar to our observations was observed in a simulation that approximated the behavior of a 2D foam at zero temperature [19]; in fact, these data are in good agreement with ours when we scale the characteristic foam size to the size of our colloidal particles, see Fig. 4(b). This similarity in the observations suggests that the behavior might be quite general for shear-melted glassy samples.

To account for our results with an approximately linear dependence of the diffusion constant with shear rate, we consider an analogy to the Stokes-Einstein expression for an equilibrium fluid, $D=k_{B} T / 6 \pi \mu R$, which is the ratio of the thermal energy to the friction factor, where $\mu$ is the viscosity. To describe the behavior of the diffusion constant of the shear-melted samples, we replace the thermal energy term by the shear energy, given by $\frac{4 \pi}{3} R_{s}{ }^{3} \eta \dot{\gamma}$, where $R_{s}$ is the radius of a characteristic volume and $\eta$ is the suspension viscosity. The friction factor is then $6 \pi \eta R_{s}$ and the diffusion coefficient is $D=\frac{2}{9} R_{s}^{2} \dot{\gamma}$. This expression is consistent with the linear dependence of $\dot{\gamma}$ observed in the data. Moreover, we obtain quantitative agreement between this expression and the data by using $R_{s}=1.8 R$, as shown by the dashed line in Fig. 4(b). The characteristic radius $R_{s}$ corresponds to a volume containing about 3-4 particles, in excellent accord with the direct measurements of the average size of the dynamic clusters.

Our results suggest that a shear-melted glass exhibits many similarities to a colloidal suspension that has been fluidized by reduction of its volume fraction or a glass that has been fluidized by increase of its temperature. We observe the signatures of dynamic heterogeneities, but only at small strains; at larger strains, these are averaged out and the particle motion becomes effectively homogeneous. We find that particles move cooperatively involving on average 3 particles. Remarkably, we can describe the diffusion behavior observed at large strains in the shearmelted glass with a modified Stokes-Einstein expression. This intriguing result supports the possibility that shear can be regarded as an "effective temperature" [19]. However, whether a highly driven nonequilibrium system can be described with concepts from equilibrium statistical mechanics needs further investigation.

We thank D. R. Reichman, F. C. MacKintosh, and A. J. Liu for helpful discussions. This work was supported by the NSF (DMR-0602684), the Harvard MRSEC (DMR-
0820484), the Deutsche Forschungsgemeinschaft (C.E.), and by the Hans Werthén, the Wennergren, and the Knut and Alice Wallenberg Foundations (J. M.).

*Present address: Liquid Crystal Institute and CPIP, Kent State University, Kent, OH 44242, USA.

†Present address: Department of Physics and Astronomy, University of Leeds, Leeds LS2 9JT, United Kingdom.

[1] W. van Megen and S. M. Underwood, Phys. Rev. Lett. 70, 2766 (1993).

[2] W. K. Kegel and A. van Blaaderen, Science 287, 290 (2000).

[3] E. R. Weeks, J. C. Crocker, A. C. Levitt, A. Schofield, and D. A. Weitz, Science 287, 627 (2000); E. R. Weeks and D. A. Weitz, Phys. Rev. Lett. 89, 095704 (2002).

[4] G. Petekidis, A. Moussaid, and P. N. Pusey, Phys. Rev. E 66, 051402 (2002); G. Petekidis, D. Vlassopoulos, and P. N. Pusey, Faraday Discuss. 123, 287 (2003); K. N. Pham, G. Petekidis, D. Vlassopoulos, S. U. Egelhaaf, P. N. Pusey, and W.C.K. Poon, Europhys. Lett. 75, 624 (2006).

[5] A. J. Liu and S. R. Nagel, Nature (London) 396, 21 (1998).

[6] M. Fuchs and M.E. Cates, Phys. Rev. Lett. 89, 248304 (2002); K. Miyazaki and D. R. Reichman, Phys. Rev. E 66, 050501(R) (2002); K. Miyazaki, H. M. Wyss, D. A. Weitz, and D. R. Reichman, Europhys. Lett. 75, 915 (2006); H. M. Wyss, K. Miyazaki, J. Mattsson, Z. Hu, D. R. Reichman, and D. A. Weitz, Phys. Rev. Lett. 98, 238303 (2007).

[7] A. Yethiraj and A. van Blaaderen, Nature (London) 421, 513 (2003).

[8] J.F. Brady and J.F. Morris, J. Fluid Mech. 348, 103 (1997).

[9] D. J. Pine, J. P. Gollub, J. F. Brady, and A. M. Leshansky, Nature (London) 438, 997 (2005).

[10] R. Besseling, E. R. Weeks, A. B. Schofield, and W. C. K. Poon, Phys. Rev. Lett. 99, 028301 (2007).

[11] G. I. Taylor, Proc. R. Soc. A 219, 186 (1953); 223, 446 (1954).

[12] K. N. Pham, G. Petekidis, D. Vlassopoulos, S. U. Egelhaaf, W.C. K. Poon, and P.N. Pusey, J. Rheol. 52, 649 (2008).

[13] P. Chaudhuri, L. Berthier, and W. Kob, Phys. Rev. Lett. 99, 060604 (2007).

[14] J. C. Crocker, M. T. Valentine, E. R. Weeks, T. Gisler, P. D. Kaplan, A. G. Yodh, and D. A. Weitz, Phys. Rev. Lett. 85, 888 (2000).

[15] P. Schall, D. A. Weitz, and F. Spaepen, Science 318, 1895 (2007).

[16] M. H. G. Krüger, Ph.D. thesis, University of Konstanz, 2009; M. Fuchs and M. E. Cates, J. Rheol. 53, 957 (2009).

[17] K. Miyazaki, D. R. Reichman, and R. Yamamoto, Phys. Rev. E 70, 011501 (2004).

[18] E. J. Salzman, G. Yatsenko, and K. S. Schweizer, J. Phys. Condens. Matter 20, 244129 (2008).

[19] I. K. Ono, C. S. OHern, D. J. Durian, S. A. Langer, A. J. Liu, and S. R. Nagel, Phys. Rev. Lett. 89, 095703 (2002). 